J. Clin. Chem. Clin. Biochem.

Vol. 14, 1976, pp. 361-364

\title{
Automated Amino Acid Analysis with Sensitive Fluorescence Detection
}

\author{
By M. Roth
}

\section{Laboratoire central, Hopital cantonal, 1211 Geneva 4 (Switzerland)}

(Received November 3, 1975/April 5, 1976)

Summary:The fluorigenic $o$-phthalaldehyde-mercaptoethanol reagent gives good reproducibility with a very stable baseline when applied to the automated analysis of amino acids at the nanomole level. Determination of even smaller quantities is possible; basic amino acids are then preferably eluted separately at constant $\mathrm{pH}$ (for example pH 6.0); this eliminates the baseline irregularities that occur with single column systems at high sensitivity settings. The reagent gives excellent results in the assay of small quantities of biological fluids such as blood plasma.

\section{Automatische Aminosäuren-Analyse mit empfindlichem Fluoreszenznachweis}

Zusammenfassung: Das Fluoreszenzreagenz o-Phthalaldehyd-Mercaptoethanol liefert gut reproduzierbare Resultate und eine sehr stabile Basislinie bei der automatischen Aminosäurenanalyse im Nanomolbereich. Auch noch kleinere Mengen lassen sich bestimmen; basische Aminosäuren werden dann am besten bei konstantem pH-Wert (z. B. 6,0) eluiert; dadurch werden Unregelmäßigkeiten in der Basislinie, die im Einsäulenverfahren bei höchster Empfindlichkeitseinstellung auftreten, vermieden. Das Reagenz eignet sich vorzüglich zur Analyse kleinster Mengen biologischer Flüssigkeiten wie Blutplasma.

\section{Introduction}

New fluorigenic reactions developed in recent years open interesting perspectives in the automatic analysis of amino acids. Some of them are much more sensitive than the colorimetric ninhydrin reaction; this is especially useful in new microanalytical systems operating with small particles of ion exchanger and microbore columns and in cases where only small quantities of sample are available. Among the different reagents that have been reported to give fluorescent products with amino acids $(1-8)$, the one consisting of a mixture of $o$-phthalaldehyde and 2-mercaptoethanol in alkaline buffer is particularly suitable for automatic amino acid analysis, because it combines the advantages of good solubility in water, fast rate of reaction at ordinary temperature and high sensitivity (5-7): The present paper presents a procedure using this reagent.

\section{Materials and Methods}

\section{Instruments}

Amino acid analyses were performed with a Liquimat automatic analyzer (Labotron, 8191 Gelting, W. Germany) equipped with two pumps and a $345 \times 4.5 \mathrm{~mm}$ column filled with a Durrum DC-6 strongly acidic ion exchanger. Average pumping rates were
$19 \mathrm{ml} / \mathrm{h}$ for buffers and $23 \mathrm{ml} / \mathrm{h}$ for the phthalaldehyde reagent. The detector was an Aminco fluoro-colorimeter equipped with a $70 \mu$ l quartz flow-cell $(0.2 \mathrm{~cm}$ inside diameter) and a General Electric F4T4/BL source lamp. The liquid was made to flow upwards through the cell. The primary and secondary filters were Corning 7-60 and 3-73, respectively. A W + W linear recorder (W + W Electronics, Basle) was used with sensitivity set between 2.5 and $20 \mathrm{mV}$. In a few experiments, a 3380 A Hewlett-Packard calculating integrator was also connected to the fluorimeter.

Elution buffers

The first and second buffer ( $\mathrm{pH} 3.07$ and 4.25 , respectively) contained $19.4 \mathrm{~g}$ trisodium citrate dihydrate, $1 \mathrm{~g}$ phenol, $200 \mathrm{mg}$ Brij-35, $900 \mathrm{ml}$ water, $\mathrm{HCl}$ to adjust the desired $\mathrm{pH}$, and water to make 1 litre. The third buffer contained $14.7 \mathrm{~g}$ trisodium citrate dihydrate, $37.4 \mathrm{~g} \mathrm{NaCl}, 1 \mathrm{~g}$ phenol, $200 \mathrm{mg}$ Brij-35, $900 \mathrm{ml}$ water, $\mathrm{HCl}$ to bring the $\mathrm{pH}$ to 6.0 , and double distilled water to make 1 litre.

\section{$o$-phthalaldehyde reagent}

The concentration of mercaptoethanol was higher than in the previously reported reagent (6), since this has been shown by Benson \& Hare (7) to improve the sensitivity for certain compounds. In addition to borate, disodium phosphate and sodium hydroxide were incorporated in the solution to increase its neutralizing power against the acidic eluates. To $400 \mathrm{mg}$ $o$-phthalaldehyde (No. 11452 from Merck, Darmstadt) were 0 -phthalaldehyde $5 \mathrm{ml}$ ethanol and $1 \mathrm{ml} 2$-mercaptoethanol. After dissolution, $250 \mathrm{ml}$ of buffer (containing $0.1 \mathrm{~mol} / 1$ borax, $0.3 \mathrm{~mol} / \mathrm{l}$ $\mathrm{Na}_{2} \mathrm{HPO}_{4}$ and $\mathrm{NaOH}$ to give $\mathrm{pH} 10.0$ ), $25 \mathrm{ml}$ of $1 \mathrm{~mol} / 1 \mathrm{NaOH}$ and water to make $500 \mathrm{ml}$ were added. This reagent may be kept up to three days at room temperature. For the separate analysis 
Tab. 1. Elution and regeneration programs for amino acid analysis

\begin{tabular}{llll}
\hline Solution pumped & $\begin{array}{l}\text { Temper- } \\
\text { ature }\end{array}$ & $\begin{array}{l}\text { Program } \\
1\end{array}$ & $\begin{array}{l}\text { Program } \\
2\end{array}$ \\
\hline pH 3.07 citrate buffer & $25^{\circ} \mathrm{C}$ & $8 \mathrm{~min}$ & $8 \mathrm{~min}$ \\
pH 3.07 citrate buffer & $43^{\circ} \mathrm{C}$ & $20 \mathrm{~min}$ & $20 \mathrm{~min}$ \\
pH 3.07 citrate buffer & $58^{\circ} \mathrm{C}$ & $40 \mathrm{~min}$ & $40 \mathrm{~min}$ \\
pH 4.25 citrate buffer & $58^{\circ} \mathrm{C}$ & $44 \mathrm{~min}$ & $44 \mathrm{~min}$ \\
pH 6.00 citrate buffer & $43^{\circ} \mathrm{C}$ & - & $40 \mathrm{~min}$ \\
pH 6.00 citrate buffer & $58^{\circ} \mathrm{C}$ & - & $68 \mathrm{~min}$ \\
0.2 mol/l NaOH & $58^{\circ} \mathrm{C}$ & $24 \mathrm{~min}$ & $24 \mathrm{~min}$ \\
pH 3.07 citrate buffer & $25^{\circ} \mathrm{C}$ & $32 \mathrm{~min}$ & $32 \mathrm{~min}$ \\
\hline
\end{tabular}

of basic amino acids, the $25 \mathrm{ml}$ of $1 \mathrm{~mol} / 1 \mathrm{NaOH}$ are omitted and $500 \mathrm{mg} \mathrm{Brij-35}$ are incorporated in the reagent.

\section{Reference mixture}

A Beckman solution containing $2.5 \mu \mathrm{mol} / \mathrm{ml}$ each of the following substances was used: Asp, Thr, Ser, Glu, Gly, Ala, $1 / 2$ Cys, Val, Met, Ile, Leu, Tyr, Phe, Lys, His, $\mathrm{NH}_{4}^{+}$and Arg. It was diluted before injection with an appropriate volume of citrate buffer, $\mathrm{pH} 2.2$.

\section{Procedure}

A single elution system for all amino acids has proved satisfactory for amounts $\geqslant 1$ nanomole. It includes a temperature decrease step in order to remove an ammonia plateau (due to traces of ammonia in the first and second buffers) from the lysine and histidine peaks. The program is shown in table 1 . To ensure a fast and reproducible temperature decrease, the water bath of the instrument incorporates a coil cooled by tap water. When quantities less than one nanomole are analyzed with this program, satisfactory results are still obtained for acidic and neutral amino acids, but the higher sensitivity setting causes troublesome fluctuations of the baseline during elution of basic amino acids with the third buffer, and the evaluation of these peaks becomes less precise. Separate elution of basic amino acids at constant $\mathrm{pH}$ (5.8 or 6.0$)$, on the other hand, provides a stable baseline from which the emergence of extremely small peaks is easily detected.

The flow diagram corresponds to that reported previously (6). Injections were made with the Labotron loop system, with volumes of 27 or $57 \mu 1$.

\section{Results}

Figure 1 shows the chromatographic separation of 0.5 nanomole each of the acidic and neutral amino acids from the reference mixture. Proline and cysteine, also present in the sample, are not detected. Excellent baseline stability is observed during elution with the first buffer. A sharp baseline rise appearing at $91 \mathrm{~min}$ is due to material released from the column by the second buffer. Its excitation and fluorescence characteristics are identical with those given by' amino acids or primary amines. This jump is therefore not due to ammonia, which would yield a different fluorescence (9).

Figure 2 shows the record after injection of $0.5 \mathrm{nmol}$ each of the same amino acids into a constant elution buffer stream of $\mathrm{pH} 6.0$. After $18 \mathrm{~min}$, when the major portion of the acidic and neutral amino acids have been eluted, tyrosine, phenylalanine, lysine, histidine, tryptophan and arginine appear as well separated peaks emerging from a stable baseline. The recovery of tryptophan is

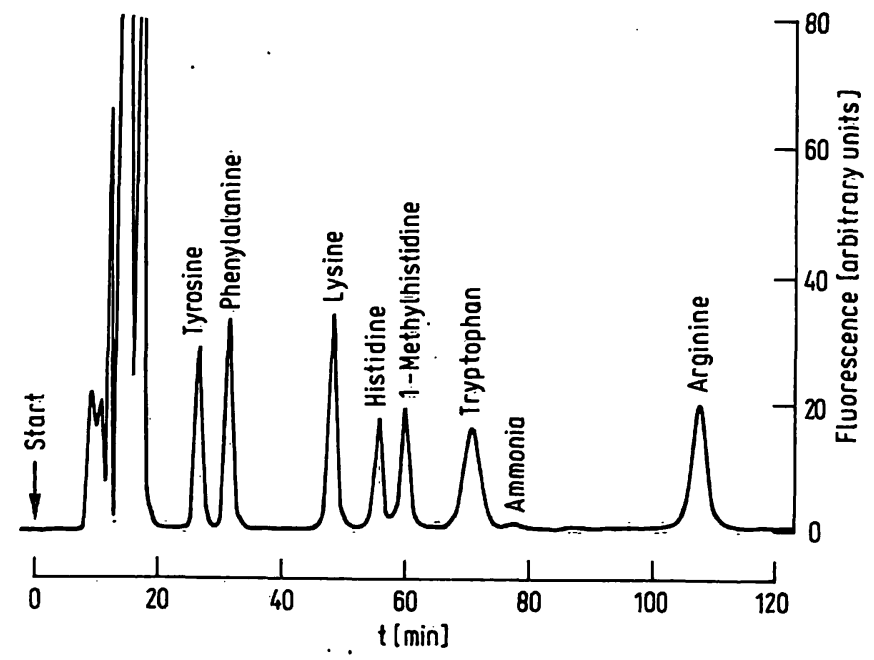

Fig. 2. Analysis of $0.5 \mathrm{nmol}$ each of aromatic and basic amino acids. Single elution buffer of $\mathrm{pH}$ 6.0. Constant temperature of $59^{\circ} \mathrm{C}$. Sample: reference mixture.

much better than in a three-buffer system, because its destruction by the more acidic buffers is avoided. Ammonia appears after $77 \mathrm{~min}$ as a small peak. The response is low because the filters do not correspond to the optimal excitation and fluorescence wavelengths shown by ammonia, which are 410 and $470 \mathrm{~nm}$, respectively (9). If one is specifically interested in ammonia, appropriate filters may be used.

The results obtained with a sample of human blood plasma are shown in Figure $3 \mathrm{a}$ and $3 \mathrm{~b}$. The first peak appearing in the chromatogram is due to sulfosalicylic acid, which was used as the deproteinizing agent. Although its excitation and fluorescence spectra are quite different from those obtained with amino acids, sufficient interfering light passed the filters to produce this peak. The figure demonstrates the good resolution 

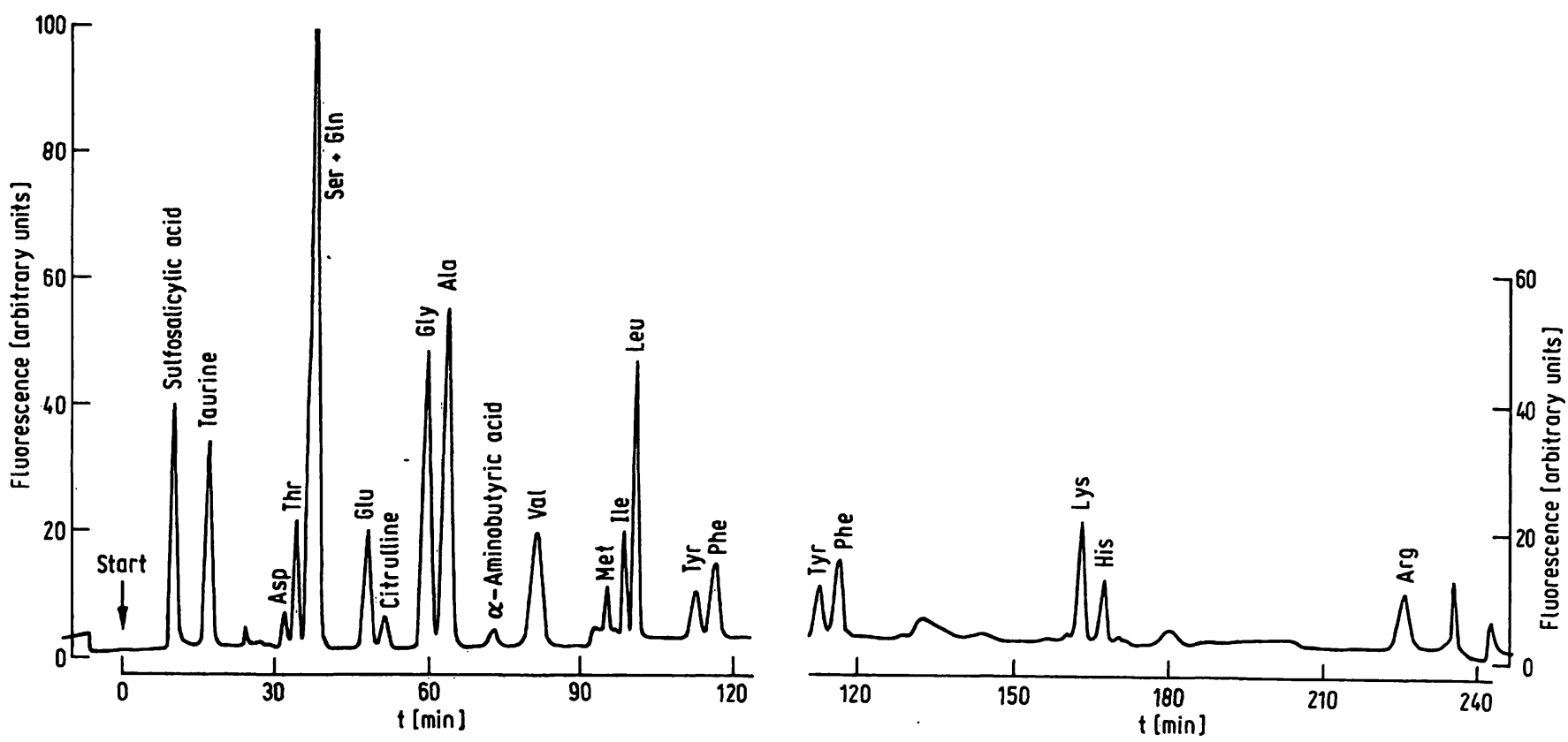

Fig. $3 \mathrm{a}$ and $3 \mathrm{~b}$. Analysis of amino acids in normal human blood plasma. To $100 \mu \mathrm{l}$ heparinized blood were added $3 \mathrm{mg}$ of sulfosalicylic acid. After mixing and centrifuging, an aliquot of the supernatant was mixed with 4 volumes of citrate buffer, $\mathrm{pH} 2.2$, and $57 \mu \mathrm{l}$ of the resulting solution were injected into the chromatograph. Single-column procedure with program No. 2.

and sensitivity achieved in the analysis of small quantities of biological samples.

Substances other than $\alpha$-amino acids, e.g. taurine, phosphoserine and phosphoethanolamine, react fairly well with the $o$-phthalaldehyde-mercaptoethanol reagent. This is also the case with $\beta$-2-thienylalanine and $\alpha$-aminof-guanidinopropionic acid which can be conveniently used as internal standards in the procedure. On the other hand, urea does not interfere.

For quantitative analysis, one can use the same principles as with systems using ninhydrin, with the advantage, however, that the response of the detector does not need to be linearized, since, within defined limits, fluorescence is proportional to concentration.

A Hewlett-Packard electronic integrator, which could be made available for a short period, was used in some experiments. It wäs not possible to perform a systematic study of the reproducibility, but the data obtained provide nevertheless an idea of the precision attainable. Thus, in two consecutive runs with 1.4 nanomole of each amino acid, differences in relative peak areas (internal standard: glutâmic acid) were: $<2 \%$ for Thr, Ala; 2-3\% for S̄er, Leu, Tyr; 3-4\% for Asp, Val, Dle, Phe; and $4-5 \%$ for Gly and Met. Seven chromatograms carried out on a shorter column $(25 \mathrm{~cm})$ with 1.4 nanomole of each amino acid gave a less satisfactory resolution, but provided relative standard deviations of the same order: alänine $1.5 \%$; serine $2.1 \%$; valine $3.2 \%$; aspartic acid $3.3 \%$ and threonine $3.6 \%$. The methionine peak must be sufficiently distinct from the preceding buffer peak, otherwise the instrument will integrate both areas together.

The fluorescence yield particular to each amino acid depends on the composition of the phthalaldehyde
Tab. 2. Relative fluorescence from amino acids as eluted with program 2 . The values are the relative integrated peak areas with glutamic acid taken as 100 . Sample: reference mixture with cysteic acid and $\beta$-2-thienylalanine added; $2 \mathrm{nmol}$ of each compound.

\begin{tabular}{lc}
\hline Substance & Relative fluorescence \\
\hline Cysteic acid & 81 \\
Aspartic acid & 97 \\
Threonine & 94 \\
Serine & 113 \\
Glutamic acid & 100 \\
Glycine & 101 \\
Alanine & 101 \\
Valine & 85 \\
Methionine & 85 \\
Isoleucine & 112 \\
Leucine & 111 \\
p-2-thienylalanine & 103 \\
Tyrosine & 80 \\
Phenylalanine & 108 \\
Lysine & 105 \\
Histidine & 109 \\
Arginine & 89 \\
\hline
\end{tabular}

reagent and the $\mathrm{pH}$ of reaction. It is established by calibration of the system with a reference mixture of amino acids. Since the final $\mathrm{pH}$ is determined by the proportions of effluent and reagent, it is essential that the flow rates be the same both for calibration and analysis of unknowns. Table 2 gives the relative fluorescence intensities provided by such a calibration run.

\section{Discussion}

The $o$-phthalaldehyde-mercaptoethanol reagent offers several advantages over ninhydrin for the automatic analysis of amino acids. Its high sensitivity permits 
precise determinations to be made at the nanomole level, and quantities of the order of 50 picomoles to be detected. Since the reaction is fast and does not require heating, the time taken by an amino acid to travel from the column to the detector is short. This prevents enlargement of the peaks by diffusion. The solubility of $o$-phthalaldehyde in water is better than that of ninhydrin, and we never experienced clogging of the tubes or the pump by the reagent.

The precision of quantitative analysis depends greatly on the stability of the baseline. Difficulties may occur with buffer peaks if they appear simultaneously with a compound to be determined. For the quantitative determination of lysine and histidine at the level of $500 \mathrm{pmol}$ or less, elution at constant $\mathrm{pH}$ therefore proves to be more convenient than stepwise $\mathrm{pH}$ gradient elution.

Since fluorescence intensity depends on the intensity of the excitation source, the stability of the lamp is essential for reproducible results. We did not observe marked changes in source intensity during single chromatographic runs. A small drift, however, is always possible. Better reproducibility may be expected from the use of a ratio fluorimeter. An internal standard should be included in every chromatogram, since the values provided by the fluorimeter are not absolute.

When applied to blood plasma, the procedure detects the same compounds as ninhydrin, except proline, hydroxyproline and cystine. The advantages are the small amount of sample required and the ease with which compounds present in only minute amounts are detected.

The good precision obtained with the $o$-phthalaldehyde reagent at the nanomole level will presumably prove very useful for the analysis of protein and peptide hydrolyzates, especially when only a few micrograms of polypeptide are available for analysis. A comparison of the $O$-phthalaldehyde reagent with fluorescamine has been made by Benson \& Hare (7). They found phthalaldehyde to be more convenient because of its solubility and stability in water.

The present disadvantage of the reagent over ninhydrin is that it does not detect proline and hydroxyproline, and that the reaction with cysteine gives only poor fluorescence. Lysine yields a good fluorescence if a surfactant like Brij is added to the buffer or the reagent $(7,10)$. The failure of cysteine to provide sufficient fluorescence may be overcome by oxidation to cysteic acid, which yields a highly fluorescent peak early in the chromatogram. Proline and hydroxyproline, if subjected to oxidation by active chlorine or $\mathrm{PbO}_{2}$, are transformed into compounds giving the fluorescence reaction. There is a problem in the control of the extent of oxidation, which should be made reproducible. Another approach which we are presently investigating uses the reagent 7-chloro4nitro-benzofurazan first described by Ghosh \& Whitehouse (1). This gives a fairly good fluorescence with proline and hydroxyproline and can be added to the effluent subsequent to the reaction with $o$-phthalaldehyde. The sensitivity achieved is superior to that with ninhydrin, although not so high as that yielded by the $o$-phthalaldehyde reagent with $\alpha$-amino acids.

One can, thus, hope that a sensitive automatic fluorimetric analysis of all protein amino acids will be possible in the near future.

\section{Acknowledgement}

I thank Miss Sylviane Jaccoud and Mrs. Mary-Claude Verlyck for their valuable assistance.

\section{References}

1. Ghosh, P. E. \& Whitehouse, M. W. (1968), Biochem. J. 108, 155-156.

2. Samejima, K., Dairman, W., Stone, J. \& Udenfriend, S. (1971), Anal. Biochem. 42, 237-247.

3. Stein, S., Böhlen, P., Stone, J., Dairman, W. \& Udenfriend, S. (1973), Arch. Biochem. Biophys. 155, 203-212.

4. Lange, H.-W., Lustenberger, N. \& Hempel, K. (1972), Z. Anal. Chem. 261, 337-342.

5. Roth, M. (1971), Anal. Chem. 43, 880-882.
6. Roth, M. \& Hampaï, A. (1973), J. Chromatogr. 83, 353 -356 .

7. Benson, J. R. \& Hare, P. E. (1975), Proc. Nat. Acad. Sci. USA 72, 619-622.

8. Maeda, M. Tsuji, A., Ganno, S. \& Onishi, Y. (1973), J. Chro matogr. 77, 434-438.

9. Taylor, S., Ninjoor, V., Dowd, D. M. \& Tappel, A. L. (1974), Anal. Biochem. 60, 153-162.

10. Schwabe, C. \& Catlin, J. C. (1974), Anal. Biochem. 61, 302-304.
PD Dr. M. Roth Laboratoire central Hôpital cantonal CH-1211 Genève 4 